\title{
Coloring axis-parallel rectangles
}

\author{
János Pach* Gábor Tardos ${ }^{\dagger}$
}

\begin{abstract}
For every $k$ and $r$, we construct a finite family of axis-parallel rectangles in the plane such that no matter how we color them with $k$ colors, there exists a point covered by precisely $r$ members of the family, all of which have the same color. For $r=2$, this answers a question of S. Smorodinsky [S06].
\end{abstract}

\section{Introduction}

Given a set of points $P$ and a family of regions $\mathcal{R}$ in the plane, in a natural way one can associate two hypergraphs with them, dual to each other. Let $H(P, \mathcal{R})$ denote the hypergraph on the vertex set $P$, whose hyperedges are all subsets of $P$ that can be obtained by intersecting $P$ with a member of $\mathcal{R}$. The hypergraph $H^{*}(P, \mathcal{R})$ is defined by swapping the roles of $\mathcal{R}$ and $P$ : its vertex set is $\mathcal{R}$, and for each $p \in P$ it has a hyperedge consisting of all regions in $\mathcal{R}$ that contain $p$.

Let $H$ be a hypergraph with vertex set $V(H)$. The chromatic number $\chi(H)$ of $H$ is the smallest number of colors in a coloring of $V(H)$ such that no hyperedge with at least two vertices is monochromatic. Let $H_{r}$ (and $H_{\geq r}$ ) denote the hypergraph on the vertex set $V(H)$, consisting of all $r$-element (at least $r$-element) hyperedges of $H$. By definition, we have $\chi(H)=\chi\left(H_{\geq 2}\right)$.

In the special case when $\mathcal{R}$ is the family of all axis-parallel rectangles and $P$ is a set of $n$ points in the plane, the problem of bounding $\chi(H(P, \mathcal{R}))$ reduces to estimating the chromatic number of the graph $G(P, \mathcal{R}):=H_{2}(P, \mathcal{R})$ consisting of all two-element (hyper)edges of $H(P, \mathcal{R})$. Krríž and Nešetřil

${ }^{*}$ City College, CUNY and Courant Institute, New York, NY, USA. Supported by NSF Grant CCF-05-14079, and by grants from NSA, PSC-CUNY, Hungarian Research Foundation OTKA, and BSF. Email: pach@cims.nyu.edu

${ }^{\dagger}$ Department of Computer Science, Simon Fraser University, Burnaby, Canada. Supported by NSERC grant 329527, and by OTKA grants T-046234, AT-048826, and NK62321. Email: tardos@cs.sfu.edu 
[KN91] gave an explicit construction showing that the chromatic number of $G(P, \mathcal{R})$ cannot be bounded by an absolute constant. Chen, Pach, Szegedy, and Tardos [CPST07] proved by a probabilistic argument that there exist $n$-element point sets $P$ such that the chromatic numbers of $G(P, \mathcal{R})$ grow as fast as at least $\Omega\left(\frac{\log n}{\log ^{2} \log n}\right)$. On the other hand, Ajwani, Elbassioni, Govindarajan, and Ray [AEGR07] proved that $\chi(H(P, \mathcal{R}))=\chi(G(P, \mathcal{R})) \leq$ $O\left(n^{383}\right)$.

It was also shown in [CPST07] that, for a fixed $r \geq 2$, a randomly and uniformly selected set $P$ of $n$ points in the unit square almost surely satisfies

$$
\chi\left(H_{r}(P, \mathcal{R})=\Omega\left(\frac{\log ^{1 /(r-1)} n}{\log ^{2} \log n}\right),\right.
$$

as $n$ tends to infinity.

Concerning the dual question, S. Smorodinsky [S06] proved that if $\mathcal{R}$ is a family of $n$ open axis-parallel rectangles and $P$ is a set of points in the plane, then $\chi\left(H^{*}(P, \mathcal{R})\right)=O(\log n)$. In other words, the rectangles in $\mathcal{R}$ can be colored by at most constant times $\log n$ colors so that, for any point $p \in P$ covered by more than one rectangle, at least two rectangles containing $p$ have different colors. Smorodinsky asked whether there always exists such a coloring with a bounded number of colors. In the present note, we answer this question in the negative, in the following stronger form

Theorem 1 Let $n \geq r \geq 2$ be integers. There exists a family $\mathcal{R}$ of $n$ axisparallel rectangles in the plane such that for any coloring of the rectangles with $k \leq C\left(\frac{\log n}{r \log r}\right)$ colors, one can find a point covered by exactly $r$ members of $\mathcal{R}$, all of the same color. Here $C>0$ is an absolute constant.

Using our notation, we have that $\chi\left(H_{r}^{*}(P, \mathcal{R})\right) \geq C\left(\frac{\log n}{r \log r}\right)$, where $P=\mathbb{R}^{2}$ (or a suitable finite subset of $\mathbb{R}^{2}$ ). For $r=2$, the above mentioned result of Smorodinsky [S06] shows that Theorem 1 is not far from being optimal.

A family of axis-parallel rectangles is said to form a $r$-fold covering of the plane if every point $p \in \mathbb{R}^{2}$ is contained in at least $r$ members of the family. It is called locally finite if no point of the plane belongs to infinitely many rectangles. We say that a covering has a $k$-split if the family can be partitioned into $k$ parts such that the union of any $k-1$ parts form a (1-fold) covering. Theorem 1 yields

Corollary 2 For every $r, k \geq 2$, there is a locally finite $r$-fold covering of the plane with axis-parallel rectangles that does not have a $k$-split. 
In the case $k=2$, Corollary 2 states that there are locally finite $r$-fold coverings of the plane with axis-parallel rectangles that cannot be partitioned into two coverings. A very simple direct construction proving this can be found in [PTT07]. Some positive results with half-planes, disks, translates of a convex polygon, etc., in the place of rectangles, were established in [P80], [MP87], [P86], [TT06], [K07], and [ACCLS07]. There is an intimate relationship between questions of this type and the notion of conflict-free colorings, introduced by Even, Lotker, Ron, and Smorodinsky [ELRS03]; see also [HS05]. Many similar problems on colorings are discussed in [BMP05] and [MP06].

\section{The construction and its basic properties}

First we have to introduce some notations.

For any two integers $c \geq 2$ and $k \geq 0$, let $[c]:=\{0,1, \ldots, c-1\}$ and let $[c]^{k}$ stand for the set of strings of length $k$ over the alphabet $[c]$. For $x \in[c]^{k}$, let $x_{j}$ denote the $j$ th digit of $x(1 \leq j \leq k)$, so that we have $x=x_{1} \ldots x_{k}$. Let $\overleftarrow{x}$ denote the reverse of $x$, that is, $\overleftarrow{x}=x_{k} \ldots x_{1}$. An initial segment of $x$ is a string $x_{1} \ldots x_{j}$ for some $0 \leq j \leq k$. Expanding $x$ as a $c$-ary fraction, we obtain a number $\bar{x}:=\sum_{j=1}^{k} x_{j} / c^{j}$.

Let $c \geq 2$ and $d \geq 1$ be integers. For any $0 \leq k \leq d, u \in[c]^{k}$, and $v \in[c]^{d-k}$, define an open axis-parallel rectangle $R_{u, v}^{k}$ in the plane as follows:

$$
R_{u, v}^{k}:=\left(\bar{u}, \bar{u}+c^{-k}\right) \times\left(\bar{v}, \bar{v}+c^{k-d}\right) .
$$

Now we are in a position to define the family of rectangles $\mathcal{R}$ meeting the requirements of Theorem 1 . Let $\mathcal{R}$ consist of the rectangles in

$$
\left\{R_{u, v}^{k} \mid 0<k<d, u \in[c]^{k}, v \in[c]^{d-k}, u_{k}=v_{d-k}\right\}
$$

together with the rectangles in

$$
\left\{R_{\varepsilon, v}^{0} \mid v \in[c]^{d}, v_{d}=0\right\} \cup\left\{R_{u, \varepsilon}^{d} \mid u \in[c]^{d}, u_{d}=0\right\},
$$

where $\varepsilon$ stands for the empty string. For convenience, we slightly change the notation. For any $z \in[c]^{d-1}, 0<k<d$, set $S_{z}^{k}:=R_{u, v}^{k}$, where $u$ is the initial segment of $z$ of length $k$, and $v$ is the initial segment of $\overleftarrow{z}$ of length $d-k$. Further, for any $z \in[c]^{d-1}$, set $S_{z}^{0}:=R_{\varepsilon, v}^{0}$ and $S_{z}^{d}:=R_{u, \varepsilon}^{d}$, where $u$ 
is obtained from $z$ by appending to it a 0 as its last digit, and $v$ is obtained from $\overleftarrow{z}$ in the same way. Using this notation, we have

$$
\mathcal{R}=\mathcal{R}(c, d)=\left\{S_{z}^{k} \mid 0 \leq k \leq d, z \in[c]^{d-1}\right\} .
$$

Clearly, we have $|\mathcal{R}|=(d+1) c^{d-1}$. Finally, let $H^{*}=H^{*}(c, d)=H^{*}\left(\mathbb{R}^{2}, \mathcal{R}(c, d)\right)$ denote the hypergraph on the vertex set $\mathcal{R}=\mathcal{R}(c, d)$, whose hyperedges are all nonempty subsets of $\mathcal{S} \subseteq \mathcal{R}$ for which there is a point in the plane covered by the elements of $\mathcal{S}$, but by no other element of $\mathcal{R}$.

The most important property of our construction is the following.

Theorem 3 Let $d>0,2 \leq r<c$, and let $H^{*}=H^{*}(c, d)$ denote the hypergraph defined above. If a subset $I \subseteq \mathcal{R}(c, d)$ contains no hyperedge of $H^{*}$ of size $r$, then we have

$$
|I| \leq \frac{c^{d-1}}{\frac{1}{r-1}-\frac{1}{c-1}}
$$

Let $G^{*}:=G^{*}(c, d)$ denote the graph $H_{2}^{*}$, consisting of all two-element hyperedges of $H^{*}=H^{*}(c, d)$. In view of Theorem 3 , the chromatic number $\chi\left(H^{*}(c, d)\right)$ satisfies the following.

Corollary 4 (1) The graph $G^{*}(2, d)$ is bipartite.

(2) The chromatic number of $G^{*}(3, d)$ is at least $\frac{d+1}{2}$.

(3) For $c \geq d+3$, the chromatic number of $G^{*}(c, d)$ is $d+1$.

Proof: To establish (1), color the rectangles $S_{z}^{k} \in \mathcal{R}(2, d)$ according to the parity of $k+\sum_{i=1}^{d} z_{i}$. Clearly, this is a proper coloring of the vertex set of $G^{*}(2, d)$.

Applying Theorem 3 with $r=2$, we obtain that the size of every independent set in $G^{*}(c, d)$ is at most $c^{d-1} /(1-1 /(c-1))$. As the total number of vertices of $G^{*}(c, d)$ is $(d+1) c^{d-1}$, the chromatic number of $G^{*}(c, d)$ is at least $(d+1)(1-1 /(c-1))$. In case $c=3$, this gives the bound claimed in (2). For $c \geq d+3$, we have $(d+1)(1-1 /(c-1))>d$, so that $\chi\left(G^{*}(c, d)\right) \geq d+1$. This is tight, since the vertices $S_{z}^{k} \in V\left(G^{*}(c, d)\right)=\mathcal{R}(c, d)$ can be colored according to the value $k, 0 \leq k \leq d$.

Theorem 1 immediately follows from

Corollary 5 Let $k, r \geq 2$ be fixed. There exists a family of $k(2 r)^{2 k r}$ axisparallel rectangles in the plane such that for any coloring of these rectangles with $k$ colors, one can find a point covered by exactly $r$ rectangles, all of which have the same color. 
Proof: Consider the family $\mathcal{R}(c, d)$ with $c=2 r, d=2 k r-1$. For any $k$-coloring of the members of $\mathcal{R}(c, d)=\mathcal{R}(2 r, 2 k r-1)$, the size of the largest color class is at least $(d+1) c^{d-1} / k=c^{d}$, which is larger than the bound in Theorem 3. Thus, the largest color class contains a hyperedge of $H^{*}(c, d)$ of size $r$. Note that the slightly smaller choices $c=2 r-1, d=2 k r-2 k$ would also suffice for the proof.

It remains to establish Theorem 3 .

\section{Proof of Theorem 3}

Let $d \geq 1$ and $c \geq 2$ be fixed. Let $\overline{\mathcal{R}}=\overline{\mathcal{R}}(c, d)$ denote the family of all rectangles $R_{u, v}^{k}$ with $0 \leq k \leq d, u \in[c]^{k}, v \in[c]^{d-k}$. Let $\bar{H}^{*}=\bar{H}^{*}(c, d)$ be the corresponding hypergraph, that is, let the vertices of $\bar{H}^{*}$ be the members of $\overline{\mathcal{R}}$, and let its hyperedges be all sets of the form $\{R \in \overline{\mathcal{R}} \mid p \in R\}$, where $p \in \mathbb{R}^{2}$.

First we study the structure of $\bar{H}^{*}$. We define $R_{u, v}^{k} \leq R_{w, z}^{l}$ if $k \leq l, u$ is an initial segment of $w$, and $z$ is an initial segment of $v$. Note that $\leq$ is a partial order on $\overline{\mathcal{R}}$. We show that the hyperedges of $\bar{H}^{*}$ form intervals in this partial order.

Lemma 6 The hyperedges of $\bar{H}^{*}$ are exactly the sets $\{e \in \overline{\mathcal{R}} \mid a \leq e \leq b\}$, where $a \leq b$ are elements of $\overline{\mathcal{R}}$.

Proof: Let $a=R_{u, v}^{k}$ and $b=R_{w, z}^{l}$ be two elements of $\overline{\mathcal{R}}$ with $a \leq b$. Let $p_{a, b}=\left(\bar{w}+c^{-l-1}, \bar{v}+c^{k-d-1}\right)$, a point of the plane. Let $e=R_{x, y}^{m} \in \overline{\mathcal{R}}$ arbitrary. We have $e=\left(\bar{x}, \bar{x}+c^{-m}\right) \times\left(\bar{y}, \bar{y}+c^{m-d}\right)$. So $p_{a, b} \in e$ if and only if $\bar{x}<\bar{w}+c^{-l-1}<\bar{x}+c^{-m}$ and $\bar{y}<\bar{v}+c^{k-d-1}<\bar{y}+c^{m-d}$. The first pair of inequalities is satisfied if and only if $m \leq l$ and $x$ is an initial segment of $w$, while the second pair is satisfied if and only if $k \leq m$ and $y$ is an initial segment of $v$. Both pairs are satisfied if and only if $a \leq e \leq b$. Therefore, the point $p_{a, b}$ shows that $\{e \in \overline{\mathcal{R}} \mid a \leq e \leq b\}$ is an edge of $\bar{H}^{*}$.

To see that $\bar{H}^{*}$ has no additional hyperedges, it is sufficient to prove the following two claims.

(1) Any two rectangles $a, b \in \overline{\mathcal{R}}$ are disjoint, unless $a \leq b$ or $b \leq a$.

(2) For any three rectangles $a \leq e \leq b$, we have $a \cap b \subseteq e$.

Here (1) shows that the vertices of any hyperedge of $\bar{H}^{*}$ is linearly ordered by $\leq$. If $a$ is the minimal vertex of a hyperedge $f$ and $b$ is its maximal vertex, then $f \subseteq\{e \in \overline{\mathcal{R}} \mid a \leq e \leq b\}$, and by (2) equality must hold.

To verify (1), let $a=R_{u, v}^{k}=a_{1} \times a_{2}$ and $b=R_{w, z}^{l}=b_{1} \times b_{2}$. Here $a_{1}$, $a_{2}, b_{1}$, and $b_{2}$ are open intervals. We may assume $k \leq l$, by symmetry. If 
for some $i \leq k$ we have $u_{i} \neq w_{i}$, then $a_{1}$ and $b_{1}$ are disjoint. If for some $i \leq d-l$, we have $v_{i} \neq z_{i}$, then $a_{2}$ and $b_{2}$ are disjoint. So if $a$ and $b$ intersect, we must have $a \leq b$. Notice that in this case we have $b_{1} \subseteq a_{1}$ and $a_{2} \subseteq b_{2}$.

To verify (2), let $a \leq e \leq b$ with $a=a_{1} \times a_{2}$ and $b=b_{1} \times b_{2}$. Using the last observation of the previous paragraph, we have $a \cap b=b_{1} \times a_{2}$ and by the same observation again, this is contained in $e$.

The type of a rectangle $R_{u, v}^{k} \in \overline{\mathcal{R}}$ is $k$. It is easy to see that if $a \leq b$ are rectangles in $\overline{\mathcal{R}}$ of type $k$ and $l$, respectively, then for all $k \leq m \leq l$, there exists precisely one $d \in \overline{\mathcal{R}}$ of type $m$ that satisfies $a \leq d \leq b$. Consider now the two-coloring of $\overline{\mathcal{R}}$, where the color of a rectangle is determined by the parity of its type. The last observation shows that in any hyperedge of $\bar{H}^{*}$ the number of vertices of the two color classes differ by at most one. In particular, no edge of size at least two is monochromatic.

Next we describe the structure of the subfamily $\mathcal{R}=\mathcal{R}(c, d)$ of $\overline{\mathcal{R}}$. The elements of $\mathcal{R}$ are partially ordered by $\leq$. Note that for any $a=S_{z}^{k}$ and $b=S_{t}^{l}$, we have $a \leq b$ if and only if $k \leq l$ and $z_{i}=t_{i}$ for all $1 \leq i \leq k$ and $l \leq i \leq d-1$. For any $a \leq b$ in $\mathcal{R}(c, d)$, we define the interval $[a, b]=\{e \in$ $\mathcal{R} \mid a \leq e \leq b\}$. For any $a=S_{z}^{k}$ and $b=S_{t}^{l}$ with $a \leq b$, the interval $[a, b]$ contains one element of type $m$ for each index $m$ such that $k \leq m \leq l$ and $z_{m}=t_{m}$. Here the type of the rectangle $S_{w}^{m}$ (inherited from $\overline{\mathcal{R}}$ ) is $m$.

Corollary 7 The hyperedges of $H^{*}(c, d)$ are exactly the intervals $[a, b]$, where $a \leq b$ are vertices of $H^{*}(c, d)$.

Proof: The hyperedges of $H^{*}(c, d)$ are the sets $e \cap \overline{\mathcal{R}}$ where $e$ is a hyperedge of $\bar{H}^{*}(c, d)$. The assertion follows from Lemma 6 .

Corollary 8 Two vertices $S_{z}^{k}$ and $S_{t}^{l}$ are connected in $G^{*}(c, d)$ by an edge if and only if

(1) $k \neq l$,

(2) for all indices $i$ strictly between $k$ and $l$, we have $z_{i} \neq t_{i}$, and

(3) for all other indices $i$, we have $z_{i}=t_{i}$.

Proof of Theorem 3: Let us fix $d \geq 1,2 \leq r<c$ and a set $I \subseteq \mathcal{R}=$ $\mathcal{R}(c, d)$ such that no edge of $H^{*}(c, d)$ of size $r$ is contained in $I$. For any vertex $a \in \mathcal{R}$ of type $0 \leq i<d$, we define the next vertex $N(a)$ of type $i+1$ as follows. Let $N\left(S_{x}^{i}\right)=S_{x}^{i+1}$ if $i=0$ or $S_{x}^{i} \in I$. If $0<i<d$ and $S_{x}^{i} \notin I$, then let $N\left(S_{x}^{i}\right)=S_{y}^{i+1}$, where $y_{j}=x_{j}$ for all indices $1 \leq j \leq d-1, j \neq i$, and $y_{i}=\left(x_{i}+s\right) \bmod c$. We choose $s$ to be the smallest positive integer 
such that $S_{y}^{i} \notin I$. In other words, we obtain $y$ from $x$ by shifting the $i$ th digit cyclically upward until we reach a value $y$ with $S_{y}^{i} \notin I$. Note that the choice $s=c$ makes $y=x$ and $S_{y}^{i} \notin I$. We call the vertex $S_{x}^{i}$ bad if $s=c$ is the minimal choice, that is, if $1 \leq i \leq d-1, S_{x}^{i} \notin I$ and $N\left(S_{x}^{i}\right)=S_{x}^{i+1}$. Clearly, $S_{x}^{i}$ is bad if and only if $S_{x}^{i} \notin I$ but $S_{y}^{i} \in I$ for all the $c-1$ strings $y$ that differ from $x$ only at the position $i$. Therefore, for the set $B$ of bad vertices, we have $|B| \leq|I| /(c-1)$.

It is easy to see that for $0 \leq i \leq d-1$, the function $N: \mathcal{R}(c, d) \rightarrow \mathcal{R}(c, d)$ is a one-to-one mapping from the vertices of type $i$ to the vertices of type $i+1$. We call a sequence $a_{0}, a_{1}, \ldots, a_{d}$ of vertices of $\mathcal{R}$ a cluster if $N\left(a_{i}\right)=a_{i+1}$ for $0 \leq i \leq d-1$. Note that $a_{i}$ is of type $i$ for every $i$. There are $c^{d-1}$ clusters (one for each $a_{0}$ of type 0 ) and they form a partition of $\mathcal{R}$ into subsets of size $d+1$.

The main observation is the following. If in a cluster $a_{0}, a_{1}, \ldots, a_{d}$ we have $a_{i}, a_{j} \in I$ for some indices $0 \leq i \leq j \leq d$, then $a_{i} \leq a_{j}$ and $\left[a_{i}, a_{j}\right]=$ $\left\{a_{k} \mid i \leq k \leq j, a_{k} \in I \cup B\right\}$. This follows readily from the definition of the relation $\leq$.

Now we use that intervals like $\left[a_{i}, a_{j}\right]$ are edges of the hypergraph $H^{*}(c, d)$ (Corollary 7), and no edge of size $r$ is contained in $I$. Therefore, a cluster contains at most $r-1$ elements of $I$ not separated by bad vertices. If there are $b$ bad vertices in a cluster, then the cluster contains at most $(r-1)(b+1)$ elements of $I$. Summing over all clusters, we conclude that

$$
|I| \leq(r-1)|B|+(r-1) c^{d-1} .
$$

Using the inequality $|B| \leq|I| /(c-1)$ and rearranging the terms, the desired bound on $|I|$ follows.

\section{Concluding remarks}

For any $c \geq 2, d \geq 1$, define a graph $G^{\prime}=G^{\prime}(c, d)$ on the vertex set $\left\{v_{z}^{k} \mid\right.$ $\left.0 \leq k \leq d, z \in[c]^{d}\right\}$ as follows. Connect two vertices $v_{z}^{k}$ and $v_{t}^{l}$ with $k \leq l$ by an edge if and only if

(1) $k<l$,

(2) for all indices $i$ with $k<i \leq l$, we have $z_{i} \neq t_{i}$, and

(3) for all other indices $i$, we have $z_{i}=t_{i}$.

Clearly, the definition of $G^{\prime}(c, d)$ is very close to the description of $G^{*}(c, d)$ given in Corollary 8. Let us compare the two graphs.

It is easy to see that $G^{\prime}$ contains a complete graph on $d+1$ vertices. For example, let $z(i)$ be a string of $i$ zeros followed by $d-i$ ones. Notice 
that the vertices $v_{z(i)}^{i}, 0 \leq i \leq d$, form a complete subgraph. Thus, the chromatic number of $G^{\prime}$ is at least $d+1$, and this is tight, as shown by the coloring that assigns color $i$ to all vertices of the form $v_{z}^{i}$. Moreover, the vertex set of $G^{\prime}$ can be partitioned into $(d+1)$-element sets so that each set induces a complete graph in $G^{\prime}$. To see this, let $x=x_{1} \ldots x_{d} \in[c]^{d}$, and for any $0 \leq i \leq d$, define $x(i) \in[c]^{d}$ as follows: let $(x(i))_{j}=x_{j}$ if $j>i$, and let $(x(i))_{j}=\left(x_{j}+1\right) \bmod c$ if $j \leq i$. Clearly, the vertices $v_{x(i)}^{i}$ induce a complete subgraph in $G^{\prime}$, and for the different choices of $x$, they form a vertex partition. This shows that no independent set in $G^{\prime}$ contains more than a fraction of $1 /(d+1)$ of the vertices.

On the other hand, the graph $G^{*}$ is triangle-free. To verify this, consider three vertices $a=S_{z}^{k}, b=S_{t}^{l}$, and $e=S_{w}^{m}$ with $k \leq l \leq m$. If $a$ and $b$ are connected by an edge in $G^{*}$, we have $k<l$ and $z_{l}=t_{l}$. Analogously, if $b$ and $e$ are connected, then $l<m$ and $t_{l}=w_{l}$. This yields that $k<l<m$ and $z_{l}=w_{l}$, which implies that $a$ and $e$ are not connected. Despite this difference, the proof of Theorem 3 was inspired by the similarity between $G^{*}$ and $G^{\prime}$, and by the simple argument above, which provides an upper bound on the size of the largest independent set in $G^{\prime}$.

The fact that $G^{*}$ is triangle-free is not merely a coincidence. Consider any family $\mathcal{S}$ of axis-parallel rectangles in the plane, and construct a graph $G=(V, E)$ on the vertex set $V=\mathcal{S}$ by connecting two rectangles if they have a point in common that is not covered by any other member of $\mathcal{S}$. Recall that $G^{*}$ was also constructed in this manner. While $G$ may contain triangles and even subgraphs isomorphic to $K_{4}$, it cannot have a complete subgraph on five vertices. Furthermore, we can partition the edge set of $G$ into two subsets $E=E_{1} \cup E_{2}$ as follows. Let us put an edge $\left\{v_{1}, v_{2}\right\} \in E$ in $E_{1}$ if the boundaries of the rectangles $v_{1}$ and $v_{2}$ cross in four points. Otherwise, put the edge $\left\{v_{1}, v_{2}\right\}$ in $E_{2}$. It is easy to see that $G_{1}:=\left(V, E_{1}\right)$ is triangle-free. It seems that $G_{1}$ is the interesting part of $G$, as the chromatic number of the graph $G_{2}:=\left(V, E_{2}\right)$ is small. Perhaps $G_{2}$ is even $\Delta$-degenerate for an appropriate absolute constant $\Delta$, that is, every subgraph of $G_{2}$ has a vertex of degree at most $\Delta$. To prove this, it would be sufficient to show that $\left|E_{2}\right| \leq \Delta|V| / 2$.

\section{References}

[AEGR07] D. Ajwani, K. Elbassioni, S. Govindarajan, and S. Ray: Conflictfree coloring for rectangle ranges using $\tilde{O}\left(n^{382}+\epsilon\right)$ colors, in: Proc. 19th 
ACM Symp. on Parallelism in Algorithms and Architectures (SPAA 07), $181-187$.

[ACCLS07] G. Aloupis, J. Cardinal, S. Collette, S. Langerman, and S. Smorodinsky: Geometric range spaces colorings (manuscript)

[BMP05] P. Brass, J. Pach, and W. Moser: Research Problems in Discrete Geometry, Springer, Berlin, 2005.

[CPST07] X. Chen, J. Pach, M. Szegedy, and G. Tardos: Delaunay graphs of point sets in the plane with respect to axis-parallel rectangles, Random Structures and Algorithms, submitted.

[ELRS03] G. Even, Z. Lotker, D. Ron, and S. Smorodinsky: Conflict-free colorings of simple geometric regions with applications to frequency assignment in cellular networks, SIAM J. Comput. 33 (2003), 94-136.

[HS05] S. Har-Peled and S. Smorodinsky: Conflict-free coloring of points and simple regions in the plane, Discrete and Computational Geometry 34 (2005), 47-70.

[K07] B. Keszegh: Weak conflict-free colorings of point sets and simple regions, in: Canad. Conf. on Computational Geometry (CCCG 2007), Ottawa, 2007.

[KN91] I. Kříž and J. Nešetřil: Chromatic number of Hasse diagrams, eyebrows and dimension, Order 8 (1991), 41-48.

[MP87] P. Mani-Levitska and J. Pach: Decomposition problems for multiple coverings with unit balls, manuscript, 1987 http://www.math.nyu.edu/ pach/publications/unsplittable.pdf.

[MP06] J. Matoušek and A. Přívětivý: The minimum independence number of a Hasse diagram, Combinatorics, Probability and Computing 15 (2006), 473-475.

[P80] J. Pach: Decomposition of multiple packing and covering, 2. Kolloquium über Diskrete Geometrie, Salzburg (1980), 169-178.

[P86] J. Pach: Covering the plane with convex polygons, Discrete and Computational Geometry 1 (1986), 73-81.

[PTT07] J. Pach, G. Tardos, and G. Tóth: Indecomposable coverings (with in: Discrete Geometry, Combinatorics and Graph Theory, The ChinaJapan Joint Conference (CJCDGCGT 2005), Lecture Notes in Computer Science 4381, Springer, 2007, 135-148.

[PT07] J. Pach and G. Tóth: Decomposition of multiple coverings into many parts, in: Proc. 23rd ACM Symposium on Computational Geometry, ACM Press, New York, 2007, 133-137. 
[S06] S. Smorodinsky: On the chromatic number of some geometric hypergraphs, in: Proc. 17th Ann. ACM-SIAM Symp. on Discrete Algorithms (SODA 2006), ACM Press, New York, 2006, 316-323.

[TT06] G. Tardos and G. Tóth: Multiple coverings of the plane with triangles, Discrete and Computational Geometry, accepted. 